\title{
Which Fracture Pattern Characteristics of Partial Articular Radial Head Fractures Are Associated With Concomitant Injuries of Elbow?
}

\author{
Jian Zhu \\ Nankai University \\ Xiangtian Deng \\ Nankai University \\ Xiaodong Cheng \\ Third Hospital of Hebei Medical University \\ Zhanchao Tan \\ Third Hospital of Hebei Medical University \\ Hongzhi Hu \\ Wuhan Union Hospital \\ Yingze Zhang ( $\sim$ zhangyingze4036@126.com ) \\ Nankai University
}

\section{Research Article}

Keywords: Radial head, fractures, fracture pattern characteristics, concomitant associated injuries, computed tomography

Posted Date: January 19th, 2021

DOI: https://doi.org/10.21203/rs.3.rs-146709/v1

License: (c) (1) This work is licensed under a Creative Commons Attribution 4.0 International License. Read Full License 


\section{Abstract}

Background: Radial head fractures are the most common fractures around the elbow and about one third of patients have associated injuries. Identification of these injuries is important, because they often dictate the treatment. While it is not clear whether the fracture pattern characteristics of radial head were correlated with associated injuries. The aim of this study was to identify whether the fracture pattern characteristics of partial articular radial head fractures are correlated with associated elbow injuries.

Methods: Patients who had a partial articular radial head fracture from 2015-2019 were retrospective analyzed. A three-dimension radial head model was created with MIMICS software to accurately measure the fracture pattern characteristics, including the size, the arc, and the location of the fragment as well as fracture comminution status and fracture fragment separation. Information of age, gender, side and associated injuries was extracted from clinical data. The patients with and without associated elbow injuries were compared. The multivariant logistic regression was used to examine the correlation between fracture pattern characteristics and associated injuries.

Results: Of the 70 patients with partial articular radial head fractures, 41 had concomitant elbow injuries. Significant differences were not found in age $₫$ gender as well as affected side and articular surface involved. Patients with associated elbow injuries were more likely to have large fragment located in anteromedial quadrant and large arc of fracture fragment. In addition, they also had more frequently to have comminuted fracture pattern and separated fragments. However, in the multiple logistic regression analysis, large fragment located in anteromedial quadrant, comminuted fracture pattern and separated fragments remained significantly associated.

Conclusions: The present study showed a large fragment located in anteromedial quadrant, comminuted fracture pattern and separated fragments are independent risk factors for elbow associated injury after radial head fractures.

\section{Introduction}

As the most common fracture of the elbow, radial head fractures account for nearly $3 \%$ of all fractures and around $33 \%$ of the elbow fractures[1,2]. Most of these fractures are caused by a fall on the palm with outstretched arm, which may cause a large displaced fragment of articular surface $[3,4]$. The radial head is essential for the function of the elbow and forearm. It serves as an important structure in maintaining stability and transferring force from the hand to the shoulder[2]. Besides, the radial head articulates with the proximal ulnar and humerus capitulum to consist of proximal radioulnar joint and humeroradial joint. Given the important role of radial head, the fractures of radial head may result in elbow stiffness, elbow movement restriction and instability. It has been agreed that conservative treatments are recommended for nondisplaced or minimally displaced radial head fractures (Mason I) and satisfactory results would be obtained[5-7]. Similarly, there is consensus that complete articular fractures (mason III) should be 
operative treatment[6-8]. To date, the optimal treatments for displaced partial articular radial head fractures (Mason II) are still disputed[9, 10].

The decision of radial head treatment is often not determined by the radial head fracture alone. It is reported that above $50 \%$ of Mason II fractures have associated injuries[11, 12], which may be an important surgical indication if they influence the elbow stability. Therefore, identification of these potential associated injuries of elbow is of great importance for treatment decision making and preoperative planning. Meanwhile, the elbow trauma could result in a variety of radial head fracture characteristics, such as different location of fracture fragment in the radial head, different comminuted status and fragment separation. However, there are few studies focused on the correlation between the radial head fracture characteristics and the associated elbow injuries[3,13,14]. Given the paucity of literature and the importance of early diagnosis, it is necessary for us to investigate the correlation between fracture pattern characteristics and concomitant associated injuries in partial articular radial head fractures. If a correlation could be identified, we think it will be helpful to expect or exclude the potential bony and soft tissue injuries of elbow and further improve care of these patients.

The aim of this study was to confirm which fracture pattern characteristics of partial articular radial head fractures were correlated with concomitant associated injuries of elbow.

\section{Materials And Methods}

\section{Patients}

In this retrospectively study, all patients with radial head fractures who underwent elbow computed tomography (CT) examination between January 2015 and December 2019 were identified. Medical records were reviewed for age of patients at time of injury, gender, side affected. The associated injuries which included fractures and elbow dislocation were identified by pre-operative CT and intraoperative examination. The clinical research ethics committee of our hospital has approved the study protocol.

\section{Inclusion and exclusion criteria}

The inclusion criteria of this study included acute injuries, individuals with skeletal maturity, partial articular radial head fractures (AO /OTA 21B2), patients with CT of the injured elbow. Exclusion criteria were: radial neck fractures, radial head fractures involving the whole head or no displacement, no available CT-scan or the slice thickness more than $1.25 \mathrm{~mm}$, biceps tuberosity being absent or unclear, obsolete fractures, neuro-muscular disorders, and pathological fracture. The injury patterns associated with radial head fractures were categorized[14] as: (1) isolated fracture without other fractures or ligaments injury; (2) elbow dislocation; (3) terrible triad injuries; (4) trans-olecranon fracture-dislocation; (5) posterior Monteggia type fracture-dislocation; (6) coronoid fracture without elbow dislocation; (7) distal humerus fractures.

\section{Modeling technique}


In order to precisely measure the diameter of radial head, the residual articular surface and the angle of fragment spanned, a 3D model of the radial head was established with CT scan. In the present study, CT scanning were performed on several different CT scanners with slice thickness between 0.62 and $1.25 \mathrm{~mm}$. All CT data were processed into the DICOM (Digital Imaging and Communications in Medicine) format by interpolation in the imaging workstation, and then loaded into MIMICS (Materialise's Interactive Medical Image Control System) software version 19.0 (Materialise; Leuven, Belgium). By using the Hounsfield unit thresholds, the bone tissues were separated from the elbow region, and stored as a Mimics object (mask). Then, the mask was subjected to 3D reconstruction and mild fairing optimization. After that, the distal humerus and ulna were removed manually with a MaskSimulate component. The retained radial head model was used to measure diameter and observe the fracture pattern characteristics, such as separated fragments and comminution or not. The comminuted fracture pattern was defined as 3 or more fragments present.

After created the radial head model, the most prominent point in the center of the biceps tuberosity was identified by turning the model and marked (Fig. 1, a). After that, the model was rotated to the maximum view of the radial head articular surface and identified the projected point of marked point of biceps tuberosity. By using the MaskMeasure component, a largest diameter circle was created based on three points at the most edge of the residual articular surface of the radial head (Fig. 1, b). According to the studies of Mahaisavariya [15] and Swieszkowski[16], the radial head was nearly circular. So, the gross radial head articular surface was calculated with the basic mathematical formula ( $\pi r^{2}$ formula).

Following that, the 3D model was converted to a 2D picture in the PNG format and imported into the Adobe Photoshop software (Adobe Corp, San Jose, CA, USA). Then, the circle was divided into four quadrants with the projected point of biceps tuberosity as 6 o'clock position. The anterolateral (AL) quadrant was defined as 12 o'clock to 3 o'clock, posterolateral (PL) quadrant as 3 o'clock to 6 o'clock, 6 o'clock to 9 o'clock as posteromedial (PM) quadrant and 9 o'clock to 12 o'clock as anteromedial (AM) quadrant. Of note, the right radial head was clockwise calculated, while the left was counter-clockwise. The position of large fragment within radial head was also documented. When the fracture fragments spanned adjacent quadrants, the larger fracture fragment was identified through measurement and percentage comparison ${ }^{6}$. The angle of radial head fracture spanned was also measured with Adobe Photoshop software (Fig. 1, c). At last, the 3-D radial head model in MIMICS software was imported into 3-Matic 11.0 (Materialise, Belgium) to measure the residual articular surface with a Mask component (Fig. 1, d).

The measurement reliability between intraobserver and interobserver was assessed with intra-class correlation coefficients (ICCs). Two observers with no knowledge of the patients' clinical information independently measured the radiologic indices. Two weeks later, one of them repeated the measurements with the patients' order being different with the first time. During the two measurements, the patients' names remained blinded all the time.

\section{Statistical methods}


The continuous variables were expressed as mean \pm SD. Categorical variables were described using absolute and relative frequencies. In order to compare clinical and radiologic factors between the groups with and without associated injuries, a bivariant analysis were performed. The Fisher exact test or chisquare test were used for qualitative variables. The Kolmogorov-Smirnov test was performed to evaluated whether continuous data were in accordance with the normal distribution. If normal distribution was present, the Student $t$ test was used for analysis, otherwise the Mann-Whitney $U$ test was used.

For further studying the correlation between all potential factors with associated elbow injuries, we performed the multivariant logistic analysis. The dependent variable was radial head fracture with associated injures and independent ones included all variables with significant differences during the bivariant analysis. As potential confounding factors, sex and age were also considered as independent variables.

All statistical analyses were conducted with the SPSS version 25.0 (SPSS Inc., Chicago, IL, USA). A pvalue of $<0.05$ was considered as statistically significant.

\section{Results}

In our study, a total of 70 patients of radial head fractures met the inclusion and exclusion criteria. The right radial head was involved in $33(47.1 \%)$ patients, while the left side was involved in $37(52.9 \%)$ patients. Of these patients ,53 were males and 17 were females, with an average age of $38.9 \pm 13.1$ years. Forty-one of the 70 patients in this study were identified as radial head fracture with associated injuries. The most common associated injury was coronoid fracture occurring in 13 patients (18.6\%). There were 11 terrible triad fracture-dislocations (15.7\%), 5 posterior Monteggia fracture-dislocations $(7.1 \%), 5$ elbow dislocations (7.1\%), 4 distal humerus fractures (5.7\%), 3 posterior olecranon fracture-dislocations (4.3\%). According to the definitions, there were 50 fractures (71.4\%) classified as comminuted fractures and 32 patients had separated fracture fragments $(45.7 \%)$.

The measurements of the radial head diameter, residual surface of the radial head facture and arc of fracture fragment showed satisfactory intraobserver and interobserver reliabilities (Table 1). Of the 70 patients, 34 large fragments were in the AL quadrant, 33 in the AM, 3 in the PM and none in the PL quadrant (Fig. 2). The average percentage of radial head fracture surface was $40.4 \%$ (8.3-65.5\%), and the average spanning angle was $161.4^{\circ}\left(73.0^{\circ}-225.9^{\circ}\right)$ from the center of the radial head. The mean residual surface of radial head fracture was $192.7 \mathrm{~mm}^{2}\left(109.3-311.9 \mathrm{~mm}^{2}\right)$ and the mean diameter of radial head was $20.3 \mathrm{~mm}(16.0-23.6 \mathrm{~mm})$. 
Table 1

Intra- and inter-observer reliability of radial head measurements

\begin{tabular}{|lllll|}
\hline Measurements & \multicolumn{2}{l}{ Intra-observer reliability } & \multicolumn{2}{l|}{ Inter-observer reliability } \\
\cline { 2 - 5 } & ICC & $95 \%$ Cl & ICC & $95 \%$ Cl \\
\hline Radial head diameter $(\mathrm{mm})$ & 0.953 & $0.926-0.971$ & 0.824 & $0.732-0.887$ \\
\hline Residual articular surface $\left(\mathrm{mm}^{2}\right)$ & 0.902 & $0.847-0.938$ & 0.873 & $0.803-0.919$ \\
\hline Arc of fracture fragment $\left({ }^{\circ}\right)$ & 0.888 & $0.826-0.929$ & 0.837 & $0.751-0.896$ \\
\hline Abbreviations: ICC Intraclass correlation coefficient, Cl Confidence interval \\
\hline
\end{tabular}

The demographic and radiological characteristics of radial head fractures with or without associated injuries was displayed in Table 2. No significant differences were observed in age, gender, sides as well as residual surface of radial head fracture and articular surface involved between the two groups. On the contrary, there were significant differences between the individuals with and without associated injuries in different variables. Specifically, in relation to our hypothesis, patients with associated injuries were more likely to have large fragment in anteromedial quadrant. Besides, the rate of comminuted fracture, larger arc of fracture fragment as well as separated fracture fragments were also higher in patients of radial head fracture associated with injuries. 
Table 2

Differences in demographic and imaging characteristics between patients with and without associated injuries.

\begin{tabular}{|c|c|c|c|}
\hline & $\begin{array}{l}\text { No associated } \\
\text { injuries } \\
(n=29)\end{array}$ & $\begin{array}{l}\text { With associated } \\
\text { injuries } \\
(n=41)\end{array}$ & value* \\
\hline Age, y(range) & $38.1 \pm 13.1(18-77)$ & $39.4 \pm 13.2(18-74)$ & 0.699 \\
\hline Male sex & $20(69.0)$ & $33(80.5)$ & 0.268 \\
\hline Side & & & 0.873 \\
\hline Right & 14(48.3) & 19(46.3) & \\
\hline Left & 15(51.7) & $22(53.7)$ & \\
\hline Fracture patterns & & & $<0.01^{\mathrm{a}}$ \\
\hline No comminuted & 15(51.7) & $5(12.2)$ & \\
\hline Comminuted & $14(48.3)$ & $36(87.8)$ & \\
\hline Quadrant of large fragment & & & $<0.01^{\mathrm{a}}$ \\
\hline AL & $22(75.9)$ & 12(29.3) & \\
\hline AM & $5(17.2)$ & $28(68.3)$ & \\
\hline PM & $2(6.9)$ & $1(2.4)$ & \\
\hline PL & 0 & 0 & \\
\hline Separated fragment & $4(13.8)$ & $28(68.3)$ & $<0.01^{\mathrm{a}}$ \\
\hline Radial head diameter (mm) & $19.80 \pm 1.87$ & $20.59 \pm 1.63$ & 0.247 \\
\hline $\begin{array}{l}\text { Residual surface of radial head fracture } \\
\left(\mathrm{mm}^{2}\right)\end{array}$ & $191.27 \pm 46.87$ & $193.74 \pm 49.83$ & 0.834 \\
\hline Articular surface involved(\%) & $38.27 \pm 10.50$ & $41.94 \pm 12.43$ & 0.186 \\
\hline Arc of fracture fragment $\left({ }^{\circ}\right)$ & $151.85 \pm 28.16$ & $168.20 \pm 28.38$ & $0.020^{\mathrm{a}}$ \\
\hline \multicolumn{4}{|l|}{ Data are documented as mean \pm SD or $n(\%)$} \\
\hline \multicolumn{4}{|c|}{ AL: anterolateral. AM: anteromedial. PM $\square$ posteromedial. PL\posterolateral. } \\
\hline \multicolumn{4}{|c|}{$\begin{array}{l}{ }^{*} \mathrm{Chi}^{2} \text { test and Fisher exact test for categorical data and the Student } \mathrm{t} \text { test and the Mann-Whitney } \mathrm{U} \\
\text { test for continuous data. }\end{array}$} \\
\hline \multicolumn{4}{|l|}{ a statistically significant $(p<0.05)$} \\
\hline
\end{tabular}


diagnosis. Given the high incidence of this damage, our data highlights the importance of being vigilant to associated injuries, especially in the case of CT scan being not available.

Many methods were used to investigate the morphology of the radial head, including caliper ruler, plain Xrays, CT, MRI and computer aided design (CAD) software[16, 23-26]. However, due to small number of subjects or non-precise measurement, different results were reported. In order to make an accurate measurement, we used a three-dimensional CT model to analysis the radial head fracture characteristics based on MIMINCS software. The widely used MIMICS software utilizes a consistent algorithm for bone identification (on CT slides), which could minimize the judgment or bias. Its advantages include easiness to use and accurate modeling function, which could provide more detailed information of periarticular fractures than CT scans, especially when CT scans are underwent in a nonstandard position in emergency room. The satisfactory intraobserver and interobserver reliability indicated that the measured parameters were reasonable. Besides, the average diameter of radial head in our study was $20.3 \mathrm{~mm}$ compared to $20.5 \mathrm{~mm}$ in the report of Mahaisavariya B [15], in which the radial head morphology was evaluated with a reverse engineering.

The most important finding of our study was that the large fragment located in anteromedial quadrant of radial head was correlation with concomitant elbow injuries. In the current study, the large fragment in the anteromedial quarter of radial head was correlated with more than 6-fold of concomitate associated injuries than that of anterolateral quarter. Given the lack of investigations addressing this factor, our finding may be more special relevant. The mechanism of fall on the outstretched hand can produce a variety of fracture patters in elbow joint. In a biomedically study [27], Amis and Miller reported that different angles of elbow flexion in the impaction injuries could cause different type of elbow fractures (e.g. distal humerus fractures, radial head fractures, coronoid fractures and olecranon fractures). Radial head fractures occurred in the range of $0^{\circ}$ to $80^{\circ}$ flexion, distal humeral fractures in the range $115^{\circ}$ to $145^{\circ}$, coronoid fractures in the arc of $0^{\circ}$ to $35^{\circ}$ flexion and always combination with radial head fractures. In another cadaveric study, Fitzpatrick M J et al[28] proved that the rotation of forearm during axial load was the primary determinant of elbow fracture-dislocation pattern. When the forearm was in pronation, axial force often caused terrible triad injuries while the isolated elbow dislocation without fractures usually occurred in supination. The different location of radial head large fragment in patients with and without concomitant associated injuries maybe caused by different position of forearm and elbow during the accidents. In other words, the patients with associated elbow injures may have a more supinated forearm and extended elbow during a fall. Unfortunately, it is difficult for patients to recall the accurate position of forearm and elbow during a fall because the accident usually happens rapidly. Further biomechanical studies are needed to verify our assumed mechanism. The advantage of having information on location of large fragment is that it would remind the physicians to be aware of associated injuries which maybe the potential causes of elbow instability and a miss diagnosis would result in significant morbidity.

The current study also supports that the comminuted fracture pattern and separated fragments of radial head are correlation to concomitant associated injuries of elbow. In the present study, the comminuted 
fracture pattern was more than 7-fold increase to concomitate associated injuries while nearly 5 -fold increase in case of separated fragments being present. The results of this study were consistent with previous publication[12, 14, 29]. In a retrospective study, Liu G et al[29] reported 17 of 20 patients with comminuted partial radial head fractures were associated with dislocation and fractures of the elbow and all patients needed operative treatment. Based on radiographic observation Rineer CA, et al [14] reported loss of cortical contact were 21 -fold increase at risk of concomitant associated elbow injures than those of cortical contact. The elbow is relatively stable due to its bony structure. Therefore, a tremendous force is required to break out the joint. The comminuted fracture or obviously displaced fragments mean that the joint should be subjected to a serious injury. It is well known that the higher the force, the more likely to cause complex elbow fracture or dislocation.

On the contrary, the results of our study showed that radial head fracture size was not associated with the concomitant injuries of the elbow. For the stability of elbow, the ligaments are more important than the radial head and the radial head was the secondary elbow stabilizer[2, 30]. In a biomechanical study, Beingessner et al [31] investigated the association between radial head fracture size and elbow stability with intact and disrupted ligaments. In their study, radial head fractures were simulated in eight unpreserved cadaveric elbow joints by sequential removing $30^{\circ}$ up to $120^{\circ}$ wedges from the anterolateral radial head, and the elbow stability was determined by measuring the valgus angulation and ulna external rotation relative to the humerus. They found that the size of radial head fracture was not significantly associated with elbow stability during active elbow motion. In our series, the average radial head fracture size in the group with concomitant associated injuries was $41.9 \%$ compared to $38.3 \%$ in group with no associated injuries. In a similar study[3], Capo et al reported the average radial head size was $42.7 \%$ in the dislocation group while $42.3 \%$ in the remained reduced group. Besides, in the present study, no evidence has been found to support the arc of the fragment were correlation to the associated injuries with radial head fracture. A prior study[3] had reported that a higher arc of fracture was a predictor of concomitant associated injuries. In our study, the similar result was found with a bivariant analysis, but when other potential factors were controlled, the arc of radial head fracture was no longer associated with concomitant associated injuries. Nevertheless, it is difficult to compare our study with prior publication. For example, the present study included more patients with isolated partial radial head fracture compared to previous studies. These findings in our study suggest that it is unreliable using the size and arc of radial head fracture to decide the presence of concomitant associated injuries.

There are several limitations in this study in addition to the respectively nature. Firstly, we could not utilize the uninjured radial head to determine the surface area and the estimated surface area basing on the $\pi r^{2}$ formula would result in some bias. Secondly, due to the difficulty of measuring distance in a 3D radial head model, we did not use the displacement of fragment as a marker to evaluate the concomitant associated injuries. Thirdly, as the most prominent area of biceps tuberosity is broad and variable, choosing it as a measurement standard may have biased our result.

Nevertheless, to the best of our knowledge, the current study is the largest and comprehensive investigation of fracture pattern characteristics and associated elbow injures in partial articular radial 
head fractures with CT scan. A total of 70 patients and 10 variables that could be correlation with the concomitant associated injuries were analyzed. The results of our study will be hopefully helpful for the treating surgeons in the initial consultation and operative decision-making.

\section{Conclusion}

In conclusion,the present study has found that a large fragment within the anteromedial quadrant of radial head is associated with concomitant elbow injuries while the fracture size is not correlated to associated injuries. In addition, the correlation of associated elbow injuries and the comminuted fracture pattern and separated fragments of radial head were further confirmed with CT scan. These findings should caution the physicians to consider ruling out associated elbow injury according to the radial head fracture patterns.

\section{Abbreviations}

CT: Computed tomography; DICOM: Digital imaging and communications in medicine; MIMICS: Materialise's interactive medical image control system; AL: anterolateral; PL: posterolateral (PL); PM: posteromedial (PM); AM: anteromedial; ICCs: intra-class correlation coefficients; MRI: Magnetic resonance imaging; CAD: Computer aided design.

\section{Declarations}

\section{Ethics approval and consent to participate}

The study has been performed in accordance with the ethical standards in the 1964 Declaration of Helsinki, and was approved by the Committee on Ethics and the Institutional Review Board of the Third Hospital of Hebei Medical University (NO 2020-040-01). Informed consent was obtained from all individual participants included in the study.

\section{Consent to Publish}

Consent to publish was obtained from the patient detailed in this study.

\section{Availability of data and materials}

The data and materials contributing to this article may be made available upon request by sending an email to the first author.

\section{Competing Interests}

All the authors declare that they have no conflict of interest with any organization that sponsored the research. 


\section{Funding}

There is no funding received.

\section{Authors' Contributions}

Yingze Zhang designed the study; Jian Zhu and Xiangtian Deng searched relevant studies; Xiaodong Chen and Zhanchao Tan analysed and interpreted the data; Jian Zhu and Hongzhi Hu wrote the manuscript and Yingze Zhang approved the final version of the manuscript.

\section{Acknowledgements}

We appreciate the contribution of all patients, their families, the investigators, and the medical staff. We are grateful to all authors.

\section{References}

1. Lapner M, King GJ. Radial head fractures. J Bone Joint Surg Am.2013; 95(12): 1136-1143.

2. Morrey BF, Tanaka S, An KN. Valgus stability of the elbow. A definition of primary and secondary constraints. Clin Orthop Relat Res.1991; 265): 187-195.

3. Capo JT, Shamian B, Francisco R, Tan V, Preston JS, Uko L, Yoon RS, Liporace FA. Fracture pattern characteristics and associated injuries of high-energy, large fragment, partial articular radial head fractures: a preliminary imaging analysis. J Orthop Traumatol.2015; 16(2): 125-131.

4. Guitton TG, van der Werf HJ, Ring D. Quantitative three-dimensional computed tomography measurement of radial head fractures. J Shoulder Elbow Surg.2010; 19(7): 973-977.

5. Liow RY, Cregan A, Nanda R, Montgomery RJ. Early mobilisation for minimally displaced radial head fractures is desirable. A prospective randomised study of two protocols. Injury.2002; 33(9): 801-806.

6. Pike JM, Athwal GS, Faber KJ, King GJ. Radial head fractures-an update. J Hand Surg Am.2009; 34(3): 557-565.

7. Struijs PA, Smit G, Steller EP. Radial head fractures: effectiveness of conservative treatment versus surgical intervention. A systematic review. Arch Orthop Trauma Surg.2007; 127(2): 125-130.

8. Chen AC, Chou YC, Weng CJ, Cheng CY. Long-term outcomes of modular metal prosthesis replacement in patients with irreparable radial head fractures. J Orthop Surg Res.2018; 13(1): 134.

9. Athwal GS, King GJ. Partial articular fracture of the radial head. J Hand Surg Am.2010; 35(10): 16791680.

10. Shi X, Pan T, Wu D, Cai N, Chen R, Li B, Zhang R, Zhou C, Pan J. Effect of different orientations of screw fixation for radial head fractures: a biomechanical comparison. J Orthop Surg Res.2017; 12(1): 143.

11. van Riet RP, Morrey BF. Documentation of associated injuries occurring with radial head fracture. Clin Orthop Relat Res.2008; 466(1): 130-134. 
12. van Riet RP, Morrey BF, O'Driscoll SW, Van Glabbeek F. Associated injuries complicating radial head fractures: a demographic study. Clin Orthop Relat Res.2005; 441(351-355.

13. Beingessner DM, Dunning CE, Beingessner CJ, Johnson JA, King GJ. The effect of radial head fracture size on radiocapitellar joint stability. Clin Biomech (Bristol, Avon).2003; 18(7): 677-681.

14. Rineer CA, Guitton TG, Ring D. Radial head fractures: loss of cortical contact is associated with concomitant fracture or dislocation. J Shoulder Elbow Surg.2010; 19(1): 21-25.

15. Mahaisavariya B, Saekee B, Sitthiseripratip K, Oris P, Tongdee T, Bohez EL, Vander Sloten J. Morphology of the radial head: a reverse engineering based evaluation using three-dimensional anatomical data of radial bone. Proc Inst Mech Eng H.2004; 218(1): 79-84.

16. Swieszkowski W, Skalski K, Pomianowski S, Kedzior K. The anatomic features of the radial head and their implication for prosthesis design. Clin Biomech (Bristol, Avon).2001; 16(10): 880-887.

17. Martin DP, Wilt ZT, Cantlon MB, Wang ML. Controversies Surrounding the Management of the Isolated Type-II Radial-Head Fracture. JBJS Rev.2017; 5(12): e3.

18. Couture A, Hébert-Davies J, Chapleau J, Laflamme GY, Sandman E, Rouleau DM. Factors affecting outcome of partial radial head fractures: A Retrospective Cohort Study. Orthop Traumatol Surg Res.2019; 105(8): 1585-1592.

19. Itamura J, Roidis N, Mirzayan R, Vaishnav S, Learch T, Shean C. Radial head fractures: MRI evaluation of associated injuries. J Shoulder Elbow Surg.2005; 14(4): 421-424.

20. Kaas L, van Riet RP, Vroemen JP, Eygendaal D. The epidemiology of radial head fractures. J Shoulder Elbow Surg.2010; 19(4): 520-523.

21. Kaas L, van Riet RP, Vroemen JP, Eygendaal D. The incidence of associated fractures of the upper limb in fractures of the radial head. Strategies Trauma Limb Reconstr.2008; 3(2): 71-74.

22. Kodde IF, Kaas L, van Es N, Mulder PG, van Dijk CN, Eygendaal D. The effect of trauma and patient related factors on radial head fractures and associated injuries in 440 patients. BMC Musculoskelet Disord.2015; 16(135.

23. Beredjiklian PK, Nalbantoglu U, Potter HG, Hotchkiss RN. Prosthetic radial head components and proximal radial morphology: a mismatch. J Shoulder Elbow Surg.1999; 8(5): 471-475.

24. Guitton TG, van der Werf HJ, Ring D. Quantitative measurements of the volume and surface area of the radial head. J Hand Surg Am.2010; 35(3): 457-463.

25. Itamura JM, Roidis NT, Chong AK, Vaishnav S, Papadakis SA, Zalavras C. Computed tomography study of radial head morphology. J Shoulder Elbow Surg.2008; 17(2): 347-354.

26. Van Riet RP, Van Glabbeek F, Neale PG, Bimmel R, Bortier H, Morrey BF, O'Driscoll SW, An KN. Anatomical considerations of the radius. Clin Anat.2004; 17(7): 564-569.

27. Amis AA, Miller JH. The mechanisms of elbow fractures: an investigation using impact tests in vitro. Injury.1995; 26(3): 163-168.

28. Fitzpatrick MJ, Diltz M, McGarry MH, Lee TQ. A new fracture model for "terrible triad" injuries of the elbow: influence of forearm rotation on injury patterns. J Orthop Trauma.2012; 26(10): 591-596. 
29. Liu G, Chen E, Xu D, Ma W, Zhou L, Chen J, Pan Z. Open reduction and internal fixation with bone grafts for comminuted mason type II radial head fractures. BMC Musculoskelet Disord.2018; 19(1): 288.

30. Rao ZT, Yuan F, Li B, Ma N. Effect of elbow flexion angles on stress distribution of the proximal ulnar and radius bones under a vertical load: measurement using resistance strain gauges. $J$ Orthop Surg Res.2014; 9(60.

31. Beingessner DM, Dunning CE, Gordon KD, Johnson JA, King GJ. The effect of radial head fracture size on elbow kinematics and stability. J Orthop Res.2005; 23(1): 210-217.

\section{Figures}



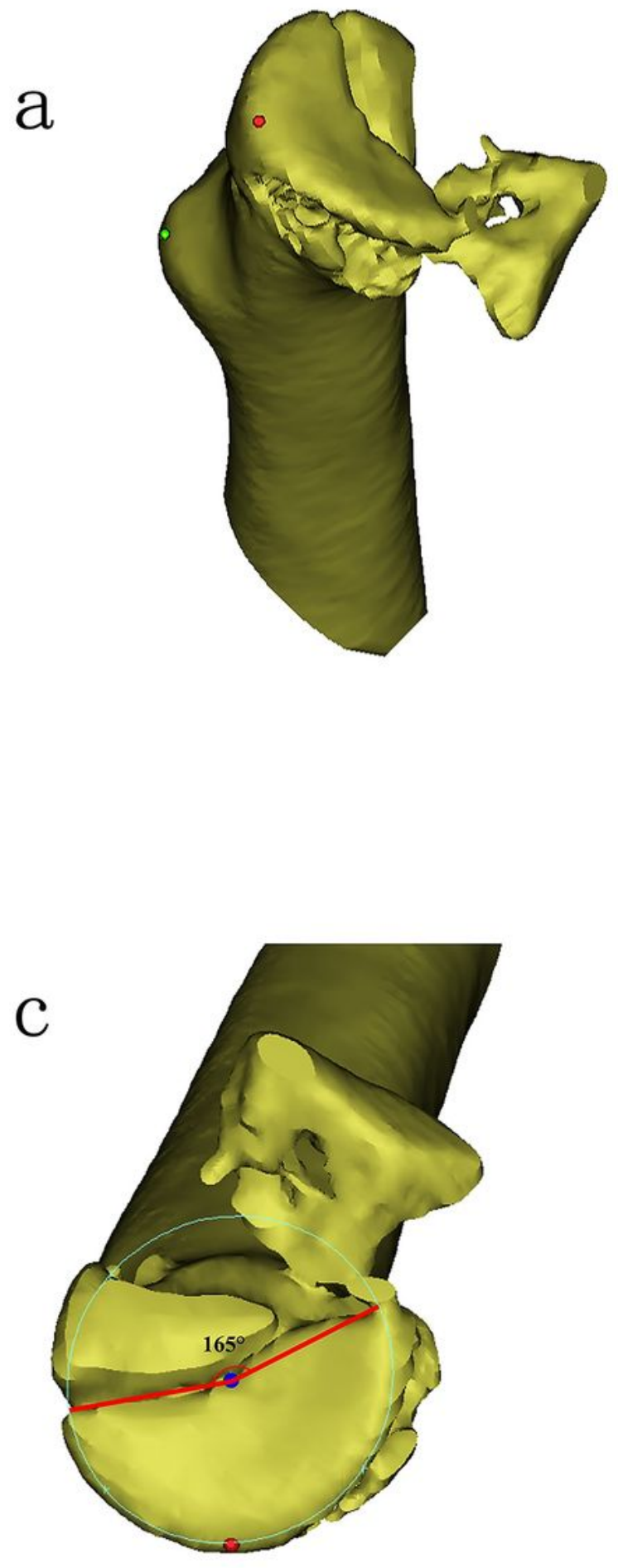

b
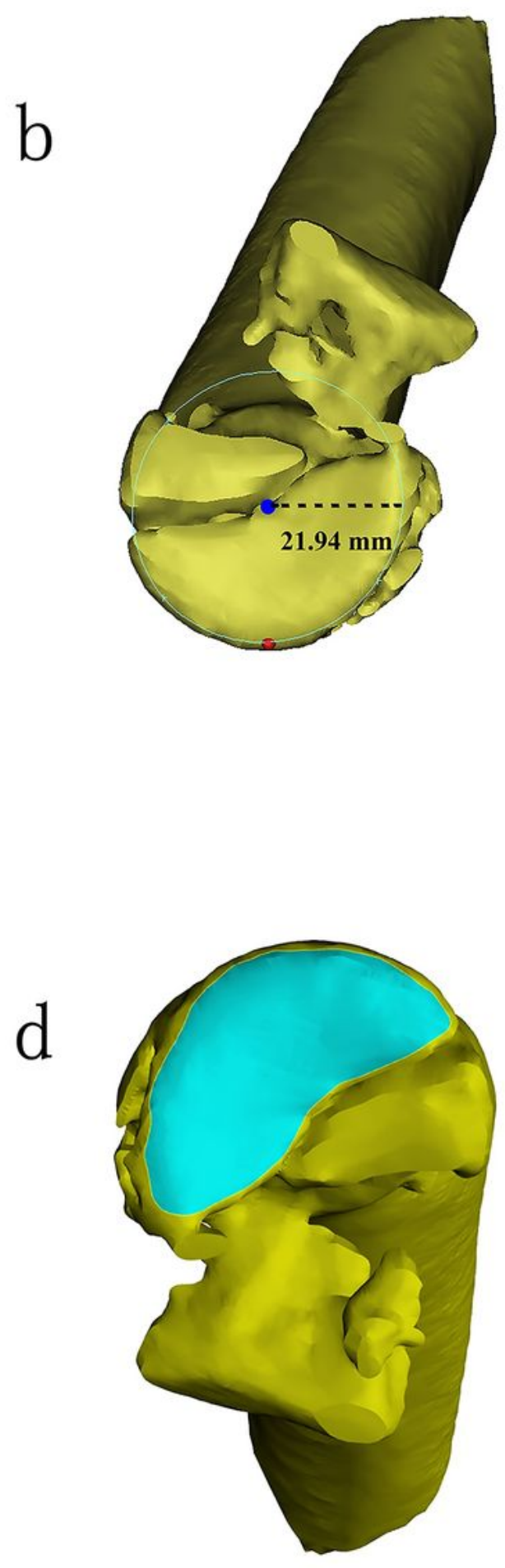

\section{Figure 1}

Screen captures are shown of radial head models within MIMICS software and Adobe Photoshop: (a) The most prominent point of the biceps tuberosity (green) is determined by turning the model and the projected point (red) of biceps tuberosity is identified. (b) A circle with largest diameter is created based on three points at the most edge of the residual articular surface of the radial head. (c) The arc of fracture 
fragment is measured in Adobe photoshop with in-bulit tools. (d) The 3-D model is imported into 3-Matic to measure the residual articular surface with a Surface component.

a

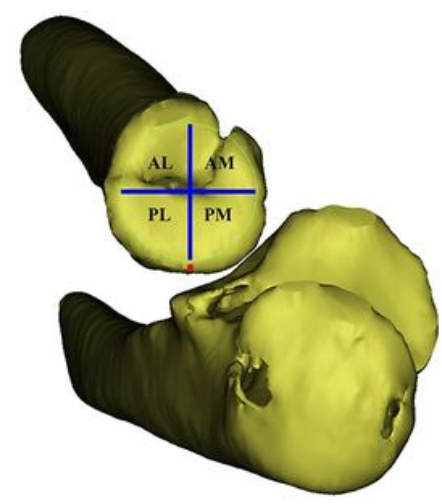

$\mathrm{b}$

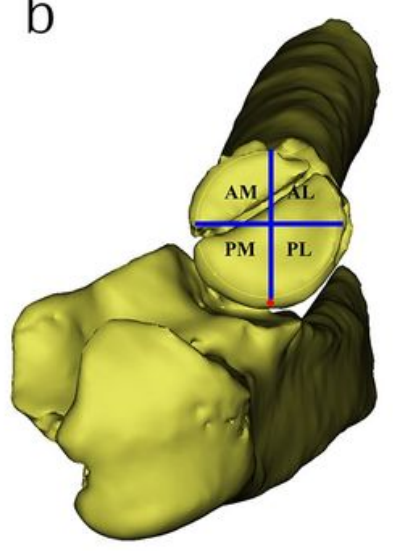

$\mathrm{C}$

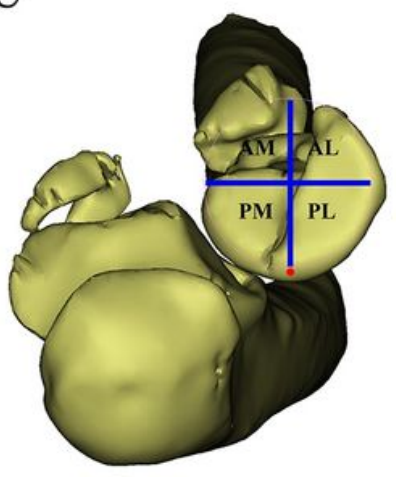

Figure 2

Top view of the radial head articular surface is showed with biceps tuberosity at 6 o'clock and the radial head is divided into 4 quadrants. (a) The large fragment is located in anteromedial quadrant; (b) The large fragment is located in anterolateral quadrant; (c) The large fragment is located in posteromedial quadrant. 